\title{
Validation of input-noise model for simulations of supercontinuum generation and rogue waves
}

\section{Frosz, Michael Henoch}

Published in:

Optics Express

Link to article, DOI:

10.1364/OE.18.014778

Publication date:

2010

Document Version

Publisher's PDF, also known as Version of record

Link back to DTU Orbit

Citation (APA):

Frosz, M. H. (2010). Validation of input-noise model for simulations of supercontinuum generation and rogue waves. Optics Express, 18(14), 14778-14787. https://doi.org/10.1364/OE.18.014778

\section{General rights}

Copyright and moral rights for the publications made accessible in the public portal are retained by the authors and/or other copyright owners and it is a condition of accessing publications that users recognise and abide by the legal requirements associated with these rights.

- Users may download and print one copy of any publication from the public portal for the purpose of private study or research.

- You may not further distribute the material or use it for any profit-making activity or commercial gain

- You may freely distribute the URL identifying the publication in the public portal

If you believe that this document breaches copyright please contact us providing details, and we will remove access to the work immediately and investigate your claim. 


\title{
Validation of input-noise model for simulations of supercontinuum generation and rogue waves
}

\author{
Michael H. Frosz \\ DTU Fotonik, Department of Photonics Engineering, Technical University of Denmark, \\ $\emptyset$ rsteds Plads 343, \\ DK-2800 Kgs. Lyngby, Denmark \\ *mhfr@fotonik.dtu.dk
}

\begin{abstract}
A new model of pump noise in supercontinuum and rogue wave generation is presented. Simulations are compared with experiments and show that the new model provides significantly better agreement than the currently ubiquitously used one-photon-per-mode model. The new model also allows for a study of the influence of the pump spectral line width on the spectral broadening mechanisms. Specifically, it is found that for four-wave mixing $(\mathrm{FWM})$ a narrow spectral line width $(\lesssim 0.1 \mathrm{~nm})$ initially leads to a build-up of FWM from quantum noise, whereas a broad spectral line width $(\gtrsim 1 \mathrm{~nm})$ initially leads to a gradual broadening of the pump spectrum. Since the new model provides better agreement with experiments and is still simple to implement, it is particularly important that it is used for future studies of the statistical properties of nonlinear spectral broadening, such as the formation of rogue waves.
\end{abstract}

(C) 2010 Optical Society of America

OCIS codes: (060.4005) Microstructured fibers; (060.4370) Nonlinear optics, fibers; (060.5295) Photonic crystal fibers; (190.4370) Nonlinear optics, fibers; (190.4380) Nonlinear optics, four-wave mixing; (190.5530) Pulse propagation and solitons.

\section{References and links}

1. M. N. Islam, G. Sucha, I. Bar-Joseph, M. Wegener, J. P. Gordon, and D. S. Chemla, "Femtosecond distributed soliton spectrum in fibers," J. Opt. Soc. Am. B 6, 1149-1158 (1989), http: //www. opticsinfobase. org/abstract. cfm?URI=josab- 6-6-1149.

2. M. H. Frosz, O. Bang, and A. Bjarklev, "Soliton collision and Raman gain regimes in continuous-wave pumped supercontinuum generation," Opt. Express 14, 9391-9407 (2006), http: / / www . opticsinfobase.org/ abstract. . f m? URI =oe-14-20-9391.

3. D. R. Solli, C. Ropers, P. Koonath, and B. Jalali, "Optical rogue waves." Nature 450, 1054-1057 (2007).

4. D. R. Solli, C. Ropers, B. Jalali, and C. Ropers, "Active control of rogue waves for stimulated supercontinuum generation," Phys. Rev. Lett. 101233902 (2008).

5. J. M. Dudley, G. Genty, and B. J. Eggleton, "Harnessing and control of optical rogue waves in supercontinuum generation,” Opt. Express 16, 3644-3651 (2008).

6. A. Mussot, A. Kudlinski, M. Kolobov, E. Louvergneaux, M. Douay, and M. Taki, "Observation of extreme temporal events in CW-pumped supercontinuum,” Opt. Express 17, 17010-17015 (2009).

7. J. M. Dudley, G. Genty, and S. Coen, "Supercontinuum generation in photonic crystal fiber," Rev. Mod. Phys. 78, 1135-1184 (2006). http: // link.aps.org/abstract/RMP/v78/p1135.

8. J. C. Travers, A. B. Rulkov, B. A. Cumberland, S. V. Popov, and J. R. Taylor, "Visible supercontinuum generation in photonic crystal fibers with a $400 \mathrm{~W}$ continuous wave fiber laser," Opt. Express 16, 14435-14447 (2008).

9. J. C. Travers, "Continuous wave supercontinuum generation" Chap. 8 in Supercontinuum generation in optical fibers, J. M. Dudley and J. R. Taylor, eds. (Cambridge University Press, 2010), ISBN 978-0-521-51480-4.

\#128386 - \$15.00 USD Received 12 May 2010; revised 10 Jun 2010; accepted 18 Jun 2010; published 25 Jun 2010

(C) 2010 OSA

5 July 2010 / Vol. 18, No. 14 / OPTICS EXPRESS 14778 
10. J. Lægsgaard, "Mode profile dispersion in the generalised nonlinear Schrödinger equation," Opt. Express 15, $16110-16123$ (2007).

11. J. C. Travers, M. H. Frosz, and J. M. Dudley, "Nonlinear fibre optics overview" Chap. 3 in Supercontinuum generation in optical fibers, J. M. Dudley and J. R. Taylor, eds. (Cambridge University Press, 2010), ISBN 9780-521-51480-4.

12. G. P. Agrawal, Nonlinear Fiber Optics (Academic Press, Burlington, MA, USA, 2007), 4th ed.

13. K. J. Blow and D. Wood, "Theoretical description of transient stimulated Raman scattering in optical fibers," IEEE J. Quantum Electron. 25, 2665-2673 (1989).

14. S. B. Cavalcanti, G. P. Agrawal, and M. Yu, "Noise amplification in dispersive nonlinear media," Phys. Rev. A 51, 4086-4092 (1995), http: / dx.doi.org/10.1103/PhysRevA. 51.4086.

15. A. Mussot, E. Lantz, H. Maillotte, T. Sylvestre, C. Finot, and S. Pitois, "Spectral broadening of a partially coherent CW laser beam in single-mode optical fibers," Opt. Express 12, 2838-2843 (2004), http : / /www . opticsexpress .org/abstract . cfm?URI=OPEX-12-13-2838.

16. J. W. Goodman, Statistical Optics (John Wiley \& Sons Inc., 2000). ISBN 0471399167.

17. J. Schröder and S. Coen, "Observation of high-contrast, fast intensity noise of a continuous wave Raman fiber laser," Opt. Express 17, 16444-16449 (2009).

18. B. E. A. Saleh and M. C. Teich, Fundamentals of Photonics (John Wiley \& Sons, Inc., New York, 1991). ISBN 0-471-83965-5.

19. P. M. Moselund, "Long-pulse supercontinuum light sources," Ph.D. thesis, DTU Fotonik, Department of Photonics Engineering, Technical University of Denmark, Oersteds Plads 343, DK-2800 Kgs. Lyngby, Denmark (2009), ISBN: 87-02062-31-8, http://orbit.dtu.dk/getResource?recordId= $251910 \& 0 b j$ ect $I d=1 \& v e r$ sion $I d=1$.

20. M. H. Frosz, P. M. Moselund, P. D. Rasmussen, C. L. Thomsen, and O. Bang, "Increasing the blue-shift of a picosecond pumped supercontinuum" Chap. 7 in Supercontinuum generation in optical fibers, J. M. Dudley and J. R. Taylor, eds. (Cambridge University Press, 2010), ISBN 978-0-521-51480-4.

21. A. Kudlinski, Personal communication (2010).

22. J. M. Dudley, G. Genty, F. Dias, B. Kibler, and N. Akhmediev, "Modulation instability, Akhmediev Breathers and continuous wave supercontinuum generation," Opt. Express 17, 21497-21508 (2009).

\section{Introduction}

Pumping a nonlinear optical fibre with low-peak power $(\lesssim 10 \mathrm{~kW}$ ), temporally long ( ps) pulses or even $\mathrm{CW}$, can lead to the formation of red-shifting solitons from random noise in the pump [1,2]. Due to similarities in the statistical properties between the solitons and oceanic rogue waves this phenomenon was later termed optical rogue waves (RWs) [3]. Optical RWs are currently receiving more and more attention, both because of fundamental curiosity to how this phenomenon is connected to e.g. oceanic RWs [3], and due to their relevance in supercontinuum generation [4-6]. The RWs are known to build up from noise in the input pulses, so any numerical studies of RWs should always ensure a physically realistic model for the input noise. However, most numerical studies use the phenomenological one-photon-permode model for representing input noise (e.g. Refs. [5-7]), even though it does not provide a realistic input spectrum (as pointed out in Refs. [2,8]), see Fig. 1. On the other hand, a highly realistic input $\mathrm{CW}$ field can be obtained by carefully modelling the build-up of lasing from quantum noise in the pump laser itself, including spectrally dependent gain, gain saturation, nonlinearity, dispersion, etc. [8,9]. The disadvantage of this approach is that is requires detailed knowledge of the inner workings of a given pump laser, and extensive modelling is required each time one wishes to model a different pump laser. Also, validation of the model by direct comparison with experimental measurements is limited.

The goal of this work is to validate an alternative input-noise model which both provides a physically reasonable input spectrum (e.g. Gaussian shaped), and at the same time is simple to implement for a given set of pump laser parameters. The new noise model is validated by comparing numerical simulations with experimentally measured supercontinuum spectra. It is found that the one-photon-per-mode model provides reasonable agreement with experiment when the pump linewidth is narrow $(0.04 \mathrm{~nm})$, but that it is necessary to include the finite linewidth of the pump in the input noise model when the pump linewidth is $\gtrsim 0.7 \mathrm{~nm}$. For large

\#128386 - \$15.00 USD Received 12 May 2010; revised 10 Jun 2010; accepted 18 Jun 2010; published 25 Jun 2010

(C) 2010 OSA

5 July 2010 / Vol. 18, No. 14 / OPTICS EXPRESS 14779 


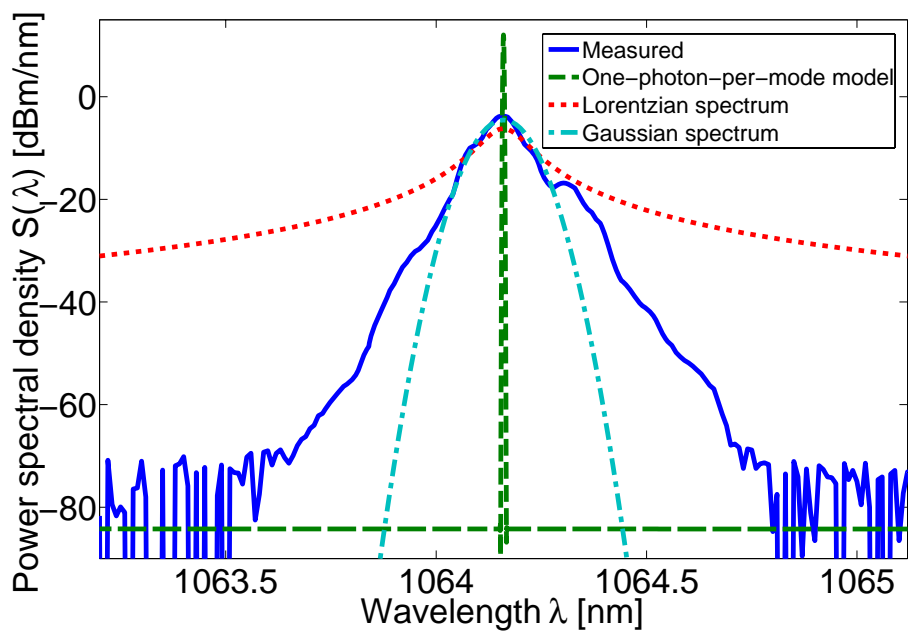

Fig. 1. Comparison of an experimentally measured spectrum from a Cheos Oy SNP-13E laser emitting $690 \mathrm{ps}$ (FWHM) pulses at $1064 \mathrm{~nm}$ with $\sim 0.1 \mathrm{~nm}$ FWHM linewidth (blue, solid); spectrum obtained from one-photon-per-mode model (green, dashed); Lorentzian power spectrum (red, dotted); Gaussian power spectrum (cyan, dash-dotted). All spectra have the same FWHM width and are normalized to have the same total power.

pump linewidth $(6 \mathrm{~nm})$ the spectral broadening is found to be initially dominated by a gradual broadening of the pump spectrum, and the contribution from quantum noise is negligible. In all cases a combination of the one-photon-per-mode model with a finite linewidth input spectrum provides the best agreement with experimental measurements.

\section{Theory}

\subsection{Propagation equation}

The propagation of an input field envelope $A(z, t)$ along the fibre axis $z$ has successfully been modelled by the so-called generalized nonlinear Schrödinger equation $[7,10,11]$ :

$$
\begin{aligned}
& \frac{\partial \tilde{C}}{\partial z}-i\left\{\beta(\omega)-\beta\left(\omega_{0}\right)-\beta_{1}\left(\omega_{0}\right)\left[\omega-\omega_{0}\right]\right\} \tilde{C}(z, \omega)+\frac{\alpha(\omega)}{2} \tilde{C}(z, \omega)= \\
& i \gamma(\omega)\left[1+\frac{\omega-\omega_{0}}{\omega_{0}}\right] \mathscr{F}\left\{C(z, t) \int_{-\infty}^{\infty} R\left(T^{\prime}\right)\left|C\left(z, T-T^{\prime}\right)\right|^{2} \mathrm{~d} T^{\prime}\right\},
\end{aligned}
$$

where $C$ is related to the Fourier transform $\tilde{A}(z, \omega)=\mathscr{F}\{A(z, t)\}$ of the field envelope $A(z, t)$ by [10]

$$
\mathscr{F}\{C(z, t)\}=\tilde{C}(z, \omega)=\left[\frac{A_{\mathrm{eff}}(\omega)}{A_{\mathrm{eff}}\left(\omega_{0}\right)}\right]^{-1 / 4} \tilde{A}(z, \omega),
$$

where $A_{\text {eff }}(\omega)$ is the frequency dependent effective mode area, and the nonlinear coefficient $\gamma(\omega)$ is given by [10]

$$
\gamma(\omega)=\frac{n_{2} n_{0} \omega_{0}}{c n_{\text {eff }}(\omega) \sqrt{A_{\text {eff }}(\omega) A_{\text {eff }}\left(\omega_{0}\right)}},
$$

where $n_{2}$ is the nonlinear-index coefficient of the waveguide material set to the value corresponding to fused silica: $n_{2}=2.6 \cdot 10^{-20} \mathrm{~m}^{2} / \mathrm{W}$ [12]. $n_{\text {eff }}(\omega)$ is the frequency dependent effective index of the guided mode and $n_{0}=n_{\mathrm{eff}}\left(\omega_{0}\right)$. The variation of $n_{\mathrm{eff}}(\omega)$ is usually much 
smaller than the variation of $A_{\text {eff }}(\omega)$ and therefore neglected in the calculation of $\gamma(\omega)$. $\omega_{0}$ is a chosen reference (angular) frequency, usually the central frequency of the pump laser. $R(t)$ is the Raman response of the nonlinear waveguide. Here the standard approximation for silica glass was used $[12,13]$ :

$$
R(t)=\left(1-f_{\mathrm{R}}\right) \delta(t)+f_{\mathrm{R}} \frac{\tau_{1}^{2}+\tau_{2}^{2}}{\tau_{1} \tau_{2}^{2}} \exp \left(-t / \tau_{2}\right) \sin \left(t / \tau_{1}\right) \Theta(t),
$$

where $\delta(t)$ is the Dirac delta function, $f_{\mathrm{R}}=0.18$ is the fractional Raman response, $\tau_{1}=12.2 \mathrm{fs}$, $\tau_{2}=32 \mathrm{fs}$, and $\Theta(t)$ is the Heaviside step function. Finally, $\alpha(\omega)$ is the wavelength dependent loss. Equation (1) is solved using the split-step Fourier method [12].

\subsection{Noise model}

A physically justified input-noise model can be obtained from a phase-diffusion model which naturally includes the spectral linewidth of the pump laser as a parameter $[2,14,15]$. However, the assumptions of the phase-diffusion model leads to a Lorentzian spectrum, which only has reasonable approximation to a typical laser spectrum over a very limited bandwidth: see Fig. 1. Since a Gaussian input spectrum would provide a much better approximation to a typical pump laser spectrum (the Gaussian fit is seen to be very good over a range of more than $20 \mathrm{~dB}$ in Fig. 1), a simple attempt to improve the phase-diffusion model is to spectrally filter the output of the phase-diffusion model, with a function that reshapes the Lorentzian spectrum into a Gaussian spectrum. This is the approach considered here, outlined in further details in the following.

The phase-diffusion model consists in using the input field envelope given by $[14,15]$

$$
A(0, T)=\sqrt{P(T)} \exp [i \delta \phi(T)]
$$

where $\delta \phi$ is a small fluctuation with zero ensemble average, $\langle\delta \phi\rangle=0 . P(T)$ is the power of the input quasi-CW (longer than picoseconds) or CW field. As described in more detail in Ref. [2] the random phase fluctuations can be seen as arising from a random fluctuation $v_{R}$ of the CW frequency $v_{0}$, so that the instantaneous frequency $v_{\mathrm{i}}$ is [16]

$$
v_{\mathrm{i}}=v_{0}+\frac{1}{2 \pi} \frac{\mathrm{d}(\delta \phi)}{\mathrm{d} t}=v_{0}+v_{\mathrm{R}}(T) .
$$

The phase fluctuation $\delta \phi(T)$ is found from [16]

$$
\delta \phi(T)=2 \pi \int_{-\infty}^{t} v_{\mathrm{R}}(\xi) \mathrm{d} \xi .
$$

$\nu_{\mathrm{R}}(T)$ is modelled as Gaussian white noise, with zero mean and variance $\sigma_{v_{\mathrm{R}}}^{2}$, where $\sigma_{v_{\mathrm{R}}}^{2}$ can be shown to be related to the FWHM spectral linewidth of the input field spectrum, $\Delta \nu_{\mathrm{FWHM}}$, by [2]

$$
\sigma_{v_{\mathrm{R}}}^{2}=\frac{\Delta v_{\mathrm{FWHM}} B}{2 \pi},
$$

where $B$ is the bandwidth of the spectral window used in the propagation simulation.

The above procedure is implemented in an algorithm (Matlab implementation freely available from the Author) which includes a random number generator to stochastically sample the frequency fluctuations $v_{\mathrm{R}}$, so that one obtains the input field envelope $A_{\mathrm{L}}(T)$ with a Lorentzian power spectrum $\left|\tilde{A}_{\mathrm{L}}(\omega)\right|^{2}$,

$$
\left|\tilde{A}_{\mathrm{L}}(\omega)\right|^{2}=P_{\mathrm{av}} \frac{\Delta v_{\mathrm{FWHM}}}{2 \pi} \frac{1}{\left(v-v_{0}\right)^{2}+\left(\Delta v_{\mathrm{FWHM}} / 2\right)^{2}},
$$

\#128386 - \$15.00 USD Received 12 May 2010; revised 10 Jun 2010; accepted 18 Jun 2010; published 25 Jun 2010

(C) 2010 OSA 5 July 2010 / Vol. 18, No. 14 / OPTICS EXPRESS 14781 
where $v_{0}$ is the center frequency of the spectrum and $P_{\mathrm{av}}$ is the average power of the quasiCW pulses or the CW field; either way $\tilde{A}_{\mathrm{L}}(\omega)$ is normalized so that $\int_{0}^{\infty}\left|\tilde{A}_{\mathrm{L}}(\omega)\right|^{2} \mathrm{~d} \omega=P_{\mathrm{av}}$. To reshape this into a Gaussian power spectrum $\left|\tilde{A}_{\mathrm{G}}(\omega)\right|^{2}$ with the same average power,

$$
\left|\tilde{A}_{\mathrm{G}}(\omega)\right|^{2}=P_{\mathrm{av}} \frac{1}{\Delta v \sqrt{\pi}} \exp \left[-\frac{\left(v-v_{0}\right)^{2}}{\Delta v^{2}}\right],
$$

where $\Delta v=\Delta v_{\mathrm{FWHM}} /[2 \sqrt{\ln (2)}]$, one can simply take the output spectrum $\tilde{A}(\omega)$ from the phase-diffusion model and multiply it with the function

$$
\begin{aligned}
& \frac{\tilde{A}_{\mathrm{G}}(\omega)}{\tilde{A}_{\mathrm{L}}(\omega)}= \\
& \frac{2[\ln (2) \pi]^{1 / 4}}{\Delta v_{\mathrm{FWHM}}} \exp \left[-\frac{1}{2} \frac{\left(v-v_{0}\right)^{2}}{\left[\Delta v_{\mathrm{FWHM}} /(2 \sqrt{\ln (2)})\right]^{2}}\right] \sqrt{\left(v-v_{0}\right)^{2}+\left(\Delta v_{\mathrm{FWHM}} / 2\right)^{2}} .
\end{aligned}
$$

It must be noted that the Lorentzian spectrum from the phase-diffusion model can be reshaped into any arbitrary spectral form using the same method presented above; one does not necessarily have to reshape it into a Gaussian shape.

It should also be noted that even when one models a CW input field by setting $P(T)$ to a constant in Eq. (5), the frequency fluctuations inherent in the phase-diffusion model lead to significant temporal intensity fluctuations of the input field after an arbitrarily small propagation length in a dispersive medium. The model therefore does include fast temporal intensity fluctuations, which are known to exist in CW lasers [17]. Also, it was confirmed that the spectral filtering outlined above leads to temporal fluctuations of the input field intensity close to the temporal coherence time of the pump expected from the relation $[9,18] \tau_{\mathrm{c}} \approx 0.66 / \Delta \nu_{\mathrm{FWHM}}$, even before propagation.

To illustrate how the choice of input noise model influences the spectral broadening, Fig. 2 shows the calculated spectra after $36 \mathrm{~cm}$ of propagation using the same parameters as in Section 3.2 , and using either the one-photon-per-mode model or the Gaussian spectrum phase-diffusion model. It is seen that when using the one-photon-per-mode model, the pump line remains narrow, and two side peaks appear on either side of the pump as a result of FWM growing from the background noise provided by the one-photon-per-mode input (seen as an almost horizontal feature of the input spectrum). When using the Gaussian input spectrum it is seen that the two side peaks do not appear at this propagation length because of too low initial spectral power at the wavelengths with maximum gain; instead, it is seen that there is a gradual broadening of the pump seeded by the finite spectral width of the pump.

Next, it is investigated whether using a finite spectral linewidth input leads to better agreement with experimental measurements, than the one-photon-per-mode model.

\section{Validation of noise model}

Simulations using either the one-photon-per-mode model, the Gaussian spectrum phasediffusion model, or a combination of the two were performed using numerical parameters chosen as close as possible to three different sets of experimental conditions. The numerically calculated spectra were then compared with the experimentally measured spectra. The experimental conditions vary significantly in many parameters, but most importantly the pump spectral linewidth varies two orders of magnitude between the experiments.

\#128386 - \$15.00 USD Received 12 May 2010; revised 10 Jun 2010; accepted 18 Jun 2010; published 25 Jun 2010

(C) 2010 OSA

5 July 2010 / Vol. 18, No. 14 / OPTICS EXPRESS 14782 


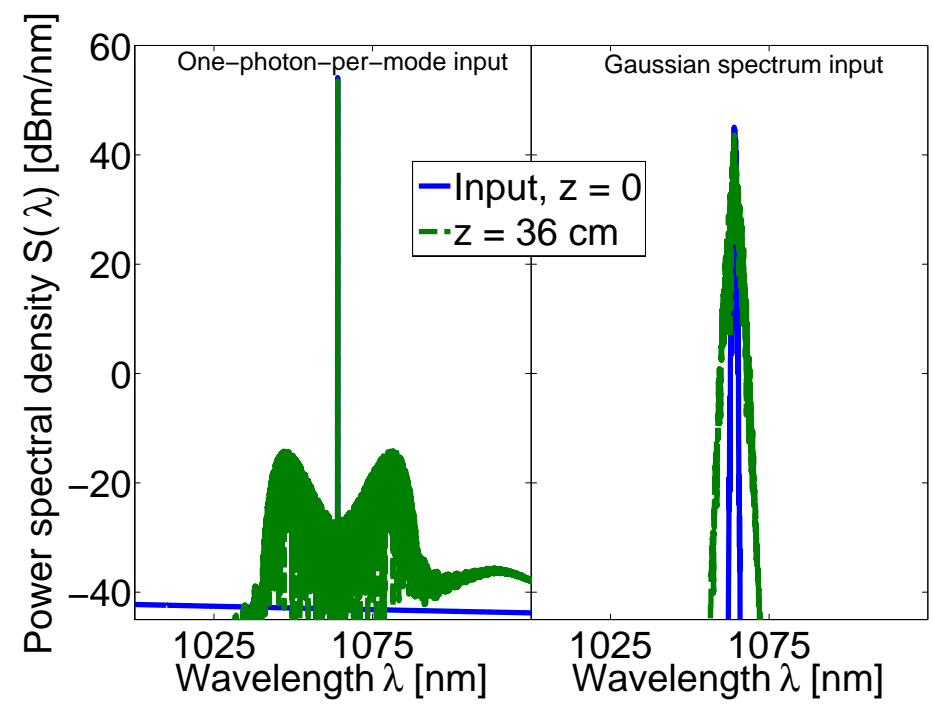

Fig. 2. Simulations of spectra after $36 \mathrm{~cm}$ of propagation (green, dashed) for the same parameters as used in Section 3.2. The input spectra are shown as blue, solid lines. Left: using the one-photon-per-mode model. Right: using the Gaussian spectrum phase-diffusion model.

\subsection{6 nm pump spectral linewidth}

The first considered experimental setup consists of using a modelocked $\mathrm{Yb}$ fibre laser amplified in several stages to obtain $6.0 \mathrm{~kW}$ peak power pulses of $14.6 \mathrm{ps}$ FWHM duration at $1066 \mathrm{~nm}$ coupled into the nonlinear fibre [19]. The pump spectral linewidth was estimated to $\sim 6 \mathrm{~nm}$ FWHM, and the nonlinear fibre was a $22 \mathrm{~cm}$ long photonic crystal fiber (PCF) with pitch $\Lambda=3.79 \mu \mathrm{m}$, and relative hole size $d / \Lambda=0.79$.

The simulated spectra using the different input noise models are shown together with an experimentally measured spectrum in Fig. 3. It is seen that the one-photon-per-mode model leads to an output spectrum much narrower than the experimentally measured spectrum. Using the Gaussian spectrum phase noise model (either with or without an added one-photon-per-mode noise background) leads to a calculated spectrum much closer to the measured spectrum. Most noteworthy, the fall-off of the spectrum on the long-wavelength side is very similar to that of the measured spectrum; this indicates that the appearance of red-shifted solitons is modelled more accurately when using the Gaussian spectrum input. The appearance of red-shifted solitons is intimately linked to rogue waves, so this is a very important advantage of using the Gaussian spectrum input model. The notable difference in the location of the short-wavelength peak (measured: $644 \mathrm{~nm}$; using Gaussian spectrum input: $770 \mathrm{~nm}$ ) can be due to deviations in the PCF structure from uniformly distributed holes with perfect circular shape. These types of deviations (e.g. somewhat elliptically shaped holes) lead to a difference in dispersion profile between the theoretically calculated and the real dispersion profile, and leads to a shift of the short-wavelength peak [20].

It should be noted that no arbitrary offset-shifting has been made of the spectra presented in Fig. 3: the experimentally measured spectrum is shifted so that integrating over the plotted spectrum equals the average output power measured experimentally; the simulated spectra are shifted so that integrating over the input spectra equals the average input power. The normalizations of the measured and the simulated spectra were therefore completely independent from each other to give the most fair and un-biased comparison.

\#128386 - \$15.00 USD Received 12 May 2010; revised 10 Jun 2010; accepted 18 Jun 2010; published 25 Jun 2010

(C) 2010 OSA

5 July 2010 / Vol. 18, No. 14 / OPTICS EXPRESS 14783 


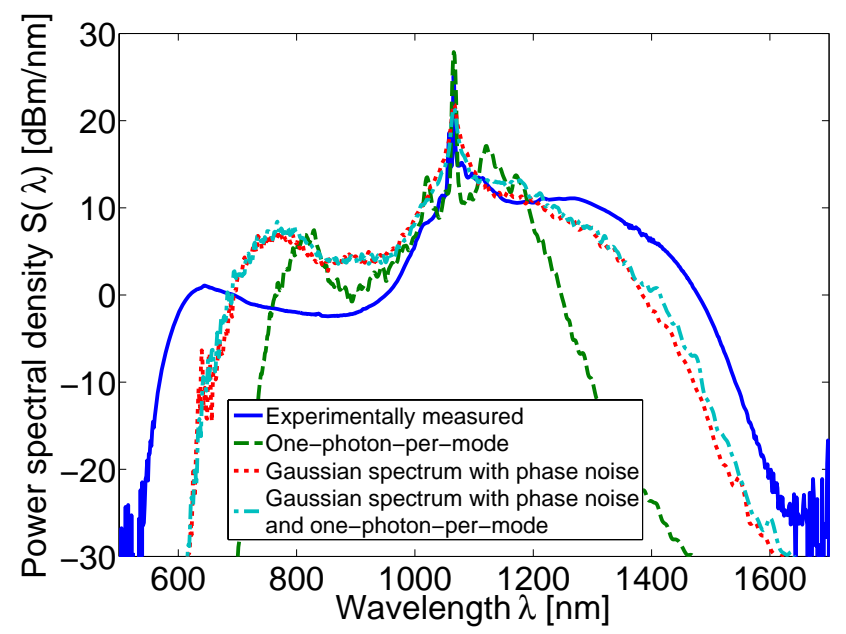

Fig. 3. Experimental measurement by P. M. Moselund [19] obtained with a $22 \mathrm{~cm}$ photonic crystal fiber pumped by $14.6 \mathrm{ps}$ pulses with $6.0 \mathrm{~kW}$ peak power (blue, solid). Corresponding simulations using either one-photon-per-mode (green, dashed), phase noise with Gaussian spectrum (red, dotted), or a combination of the two noise models (cyan, dash-dotted). The simulated spectra are obtained by averaging over 5 simulations for each type of input noise, and afterwards smoothed by convolution with a $2 \mathrm{~nm}$ wide Gaussian function.

\subsection{7 nm pump spectral linewidth}

The next considered experimental setup is presented in Ref. [6]: $10 \mathrm{~W} \mathrm{CW}$ input at $1064 \mathrm{~nm}$ with $0.7 \mathrm{~nm}$ FWHM spectral width, coupled into a $400 \mathrm{~m}$ long PCF. The PCF dispersion parameters used for the simulation were obtained from a finite element method-calculated dispersion profile based on an SEM picture of the PCF cross-section, and found to be in excellent agreement with the measured dispersion of the PCF [21]. The simulations also included an experimentally measured wavelength-dependent loss profile of the PCF [21], which turned out to be crucial to obtain good agreement between the experimentally measured and the simulated output average power.

The measured and calculated spectra are shown together in Fig. 4. In this case it is also seen that using the one-photon-per-mode model leads to too little spectral broadening of the output, and that using the Gaussian input spectrum leads to a better agreement with the experimental measurement. However, it is seen that combining the Gaussian input spectrum with the onephoton-per-mode background leads to even better agreement with experiment. This is further explored in Section 4.

Again it should be noted that the measured and calculated spectra are normalized independently using the same procedure as in the previous section to give a fair and un-biased comparison.

\subsection{04 nm pump spectral linewidth}

The final comparison is to the experimental setup described in Ref. [22]: 1 ns FWHM duration input pulses with $43 \mathrm{~W}$ peak power at $1064 \mathrm{~nm}$ and $\sim 0.04 \mathrm{~nm}(10 \mathrm{GHz})$ FWHM spectral linewidth. The PCF is $3.9 \mathrm{~m}$ long, with structural parameters $\Lambda=1.6 \mu \mathrm{m}$, and $d / \Lambda=0.875$, but the dispersion parameters are given in Ref. [7] for a reference wavelength of $835 \mathrm{~nm}$, and were therefore simply converted to apply for a reference wavelength equal to the pump wavelength

\#128386 - \$15.00 USD Received 12 May 2010; revised 10 Jun 2010; accepted 18 Jun 2010; published 25 Jun 2010

(C) 2010 OSA

5 July 2010 / Vol. 18, No. 14 / OPTICS EXPRESS 14784 


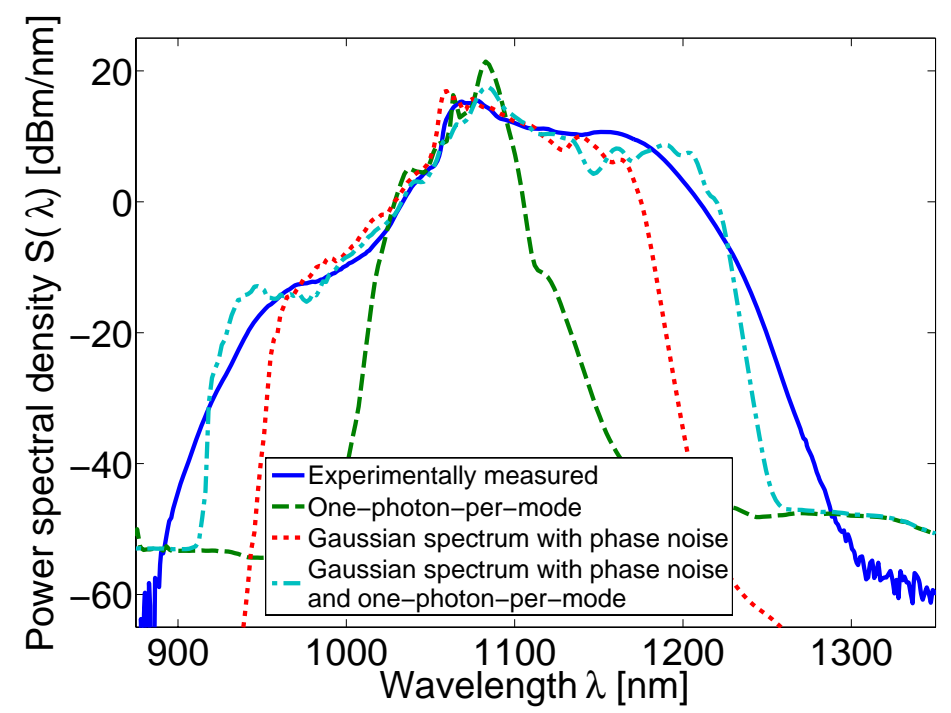

Fig. 4. Experimental measurement of output spectrum containing rogue waves, with $10 \mathrm{~W}$ CW pump and other parameters as presented in Ref. [6] (blue, solid). Simulated output spectra using either one-photon-per-mode input noise (green, dashed), Gaussian spectrum with phase noise (red, dotted), or a combination of the two noise models (cyan, dashdotted). The simulated spectra are obtained by averaging over 50 simulations for all types of input noise, and afterwards smoothed by convolution with a $1 \mathrm{~nm}$ wide Gaussian function and down-sampling to 1001 points.

used in the present case.

The measured and calculated spectra are shown together in Fig. 5. In this case the Gaussian input spectrum leads to a severe underestimation of the calculated spectral broadening, whereas the one-photon-per-mode model leads to reasonable agreement with the experiment. As in the previous two cases, the combination of Gaussian input spectrum and one-photon-per-mode background leads to the best agreement with the experiment.

The small Raman peak at $\lambda_{\mathrm{s}} \sim 1116 \mathrm{~nm}$ is clearly underestimated for all noise models. If the input power spectrum of the pump laser had a significant tail extending to $\lambda_{\mathrm{s}}$ (e.g. due to amplified spontaneous emission or Raman gain inside the pump laser), this could lead to more calculated output power at $\lambda_{\mathrm{s}}$, since the Raman peak grows as [12]

$$
\left.\left.\frac{\mathrm{d}\left|A\left(z, \lambda_{\mathrm{s}}\right)\right|^{2}}{\mathrm{~d} z} \propto \mid A\left(z, \lambda_{\mathrm{s}}\right)\right)\left.\right|^{2} \mid A\left(z, \lambda_{\mathrm{p}}\right)\right)\left.\right|^{2},
$$

where $\lambda_{\mathrm{p}}$ is the pump wavelength. A value of $\left.\mid A\left(0, \lambda_{\mathrm{s}}\right)\right)\left.\right|^{2}$ larger than that provided by the used input noise models would therefore result in an output power of the Raman peak, $\left.\mid A\left(3.9 \mathrm{~m}, \lambda_{s}\right)\right)\left.\right|^{2}$, closer to the experimentally measured. It is therefore possible that the Gaussian spectrum underestimates the spectral power at $\lambda_{\mathrm{s}}$ in this case and that using an even more realistic input spectrum than the Gaussian would give a better prediction of the size of the Raman peak.

Since the average output power was not measured for this experiment [22], it was not possible to shift the measured spectrum so that integrating over the plotted spectrum equals the average output power measured experimentally; instead an arbitrary offset has been applied. All the simulated spectra have been normalized so that integrating over the input spectra equals the average input power, assuming a repetition rate of $10 \mathrm{kHz}$.

\#128386 - \$15.00 USD Received 12 May 2010; revised 10 Jun 2010; accepted 18 Jun 2010; published 25 Jun 2010 (C) 2010 OSA 5 July 2010 / Vol. 18, No. 14 / OPTICS EXPRESS 14785 


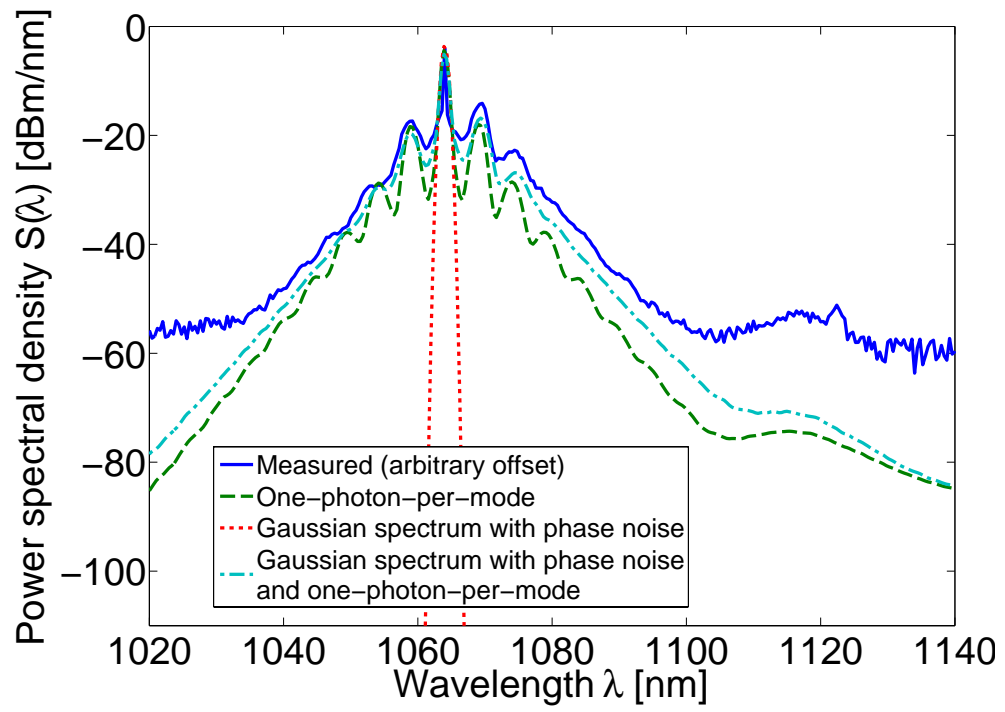

Fig. 5. Experimental measurement of output spectrum for parameters as presented in Ref. [22] (blue, solid). Simulated output spectra using either one-photon-per-mode input noise (green, dashed), Gaussian spectrum with phase noise (red, dotted), or a combination of the two noise models (cyan, dash-dotted). The simulated spectra are obtained by averaging over 10 simulations for all types of input noise, and afterwards smoothed by convolution with a $0.4 \mathrm{~nm}$ wide Gaussian function and down-sampling to 1001 points.

\section{Discussion}

The observations in the previous section can be summarized as follows.

For a narrow pump linewidth $(0.04 \mathrm{~nm})$ one obtains a reasonable agreement with experiment when using the one-photon-per-mode model. This is not surprising since the one-photon-permode model assumes an infinitesimally narrow input linewidth. Using a Gaussian input spectrum alone leads to very poor agreement with experiment for this narrow linewidth, because the FWM peaks in this case grow from the quantum noise background provided by the onephoton-per-mode model, but not by the Gaussian input spectrum alone. In this case the initial spectral broadening is therefore in principle similar to that seen in Fig. 2 (left).

For a broad pump linewidth $(6 \mathrm{~nm})$ there was found to be negligible difference between using the Gaussian input spectrum alone, or combining it with the one-photon-per-mode. This shows that in this case the spectral broadening initially grows as a gradual broadening of the pump, as in Fig. 2 (right), before the formation of red-shifting solitons. One can also view this as a case where the spectral broadening initially grows from laser noise (intensity fluctuations on the order of the coherence length), and where quantum noise has practically no influence.

For a "medium" width pump linewidth $(0.7 \mathrm{~nm})$ it was found that both quantum background noise and a Gaussian input spectrum should be included to obtain reasonable agreement with experiment. This can be explained further by comparing Figs. 2, left and right: the gradual broadening of the pump [Fig. 2 (right)] quickly leads to a spectrum overlapping with the region of gain for FWM [ 1040-1090 nm, Fig. 2 (left)]. When using only the one-photon-permode model, FWM can only grow from the quantum noise background; when combining the one-photon-per-mode model with the Gaussian input spectrum, the FWM can grow from a combination of the quantum noise and the spectral power provided by the gradual broadening of the pump. This interesting case gives a deeper understanding of the FWM-process and its

\#128386 - \$15.00 USD Received 12 May 2010; revised 10 Jun 2010; accepted 18 Jun 2010; published 25 Jun 2010 (C) 2010 OSA 5 July 2010 / Vol. 18, No. 14 / OPTICS EXPRESS 14786 
dependence on the pump spectral linewidth.

\section{Conclusions}

In all three investigated cases, a combination of the Gaussian input spectrum with one-photonper-mode background resulted in the best agreement with experimental measurements. It is therefore obvious to recommend that future investigations with supercontinuum modelling use this combined input noise model, especially since it is a simple matter of including one additional parameter (the pump spectral linewidth) in the simulations, once the algorithm is implemented (an example of a Matlab implementation of the algorithm is freely available from the Author).

The combined noise model should also be the preferred choice for future investigations of how rogue waves are formed. The rogue waves appear randomly because of the stochastic nature of the input noise, and it is therefore particularly important that one uses a noise model with the closest agreement to the physical reality.

The influence of the pump spectral linewidth on the spectral broadening process can also be examined using the combined noise model. One could also use the complete model of how laser noise arises in the pump [8,9], but that is significantly more complicated than simply including the pump spectral linewidth as an additional parameter in the simulations and using the Gaussian input spectrum combined with one-photon-per-mode noise.

Finally, it is noted that the suggested model does not require the assumption of a Gaussian input spectrum. The procedure outlined in Section 2.2 can be equally well applied to shape the input spectrum into any arbitrary shape, e.g. $\operatorname{sech}^{2}\left[\left(\omega-\omega_{0}\right) / \Delta \omega\right]$, or an experimentally measured spectrum.

\section{Acknowledgments}

The Author sincerely thanks P. M. Moselund, Technical University of Denmark and NKT Photonics A/S, A. Kudlinski, Université Lille1 in France, and J. M. Dudley, Institut FEMTO-ST in France, for providing data from the experimental measurements.

The work was financially supported by the Danish Research Council for Technology and Production Sciences (FTP), grant No. 274-07-0397.

\#128386 - \$15.00 USD Received 12 May 2010; revised 10 Jun 2010; accepted 18 Jun 2010; published 25 Jun 2010

(C) 2010 OSA 5 July 2010 / Vol. 18, No. 14 / OPTICS EXPRESS 14787 